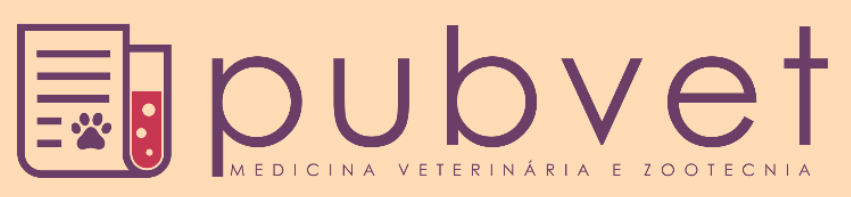

https://doi.org/10.31533/pubvet.v13n7a376.1-5

\title{
A importância da sarna sarcóptica na medicina veterinária: Revisão
}

\author{
Fernanda de Cássia Pereira Barros ${ }^{10}$, Ana Luiza Madruga de Oliveira ${ }^{10}$, Luís Eduardo \\ Monteiro Galvão $^{10}$, Leonardo Alves Farias ${ }^{2 *} *$
}

${ }^{1}$ Acadêmicos da Uninassau, Departamento de Medicina Veterinária. João Pessoa-PB Brasil.

${ }^{2}$ Orientador e docente da Uninassau (João Pessoa, PB e Brasil)-E-mail:leovetleleco@gmail.com

\begin{abstract}
Resumo. As dermatopatias parasitárias são enfermidades que representam aproximadamente $30 \%$ do total de casos atendidos em hospitais e clínicas veterinárias de animais de companhia. Uma das patologias da pele é a sarna, essa que é causada por um ácaro Sarcoptes scabiei variedade canis. Altamente contagiosa entre os animais domésticos como cães com potencial zoonótico podendo infestar o ser humano. A doença manifestase com prurido intenso na pele, formando crostas hemorrágicas, perda de pelos e provocando o aparecimento de feridas. Os exames de diagnóstico consistem em teste de arrancamento do pelo, tricograma, raspagem superficial e trial terapêutico com ectoparasiticida. Vários produtos antiparasitários vêm sendo usados para o tratamento de sarna sarcóptica que variam desde múltiplas aplicações tópicas a dose única injetável. Esta revisão objetivou verificar a importância clínica na identificação dos sinais clínicos, formas de diagnósticos e estratégias de controle parasitário apropriados e eficazes, dessa forma contribuindo para melhor proteger os cães destes parasitos. Através do presente estudo, nota-se a evidência da atenção do médico veterinário aos sinais clínicos do ácaro na clínica de pequenos animais, seu tratamento deve começar tão mediatamente quanto o diagnóstico, pois, além do fator bem-estar animal, tem-se a sarna sarcóptica que é considerada uma zoonose, sendo assim um caso de saúde pública.
\end{abstract}

Palavras chave: ácaro, cães, escabiose, sarna sarcóptica

\section{The importance of sarcoptic mange in veterinary medicine: Review}

\begin{abstract}
The skin diseases, parasitic diseases are diseases represent approximately $30 \%$ of the total number of cases seen in hospitals and veterinary clinics for pets. One of the diseases of the skin is the scab, which is cause by a mite Sarcoptes scabiei canis variety. Highly contagious among domestic animals as dogs with zoonotic potential and can infest humans. The disease manifests itself with intense itching in the skin, forming crusts hemorrhage, loss of fur and causing the appearance of sores. Diagnostic exams consist of test tricogram, superficial scraping by, and therapeutic trial with ectoparasitic. Several antiparasitic products have been use for the treatment of sarcoptic mange ranging from multiple topical applications to single-dose injectable. This review aimed to verify the clinical importance in identifying clinical signs, diagnosis and control parasitic strategies and effective, thus contributing to better protect the dogs of these parasites. Through this study, the evidence of the veterinarian's attention to clinical signs of mite in small animal clinic, your treatment should begin immediately so as the diagnosis, because, besides animal welfare factor, scabies sarcoptic which is considered a zoonosis, so a case of public health.
\end{abstract}

Keywords: mite, dogs, scabies, sarcoptic mange 


\title{
La importancia de la sarna sarcóptica em la medicina veterinaria: Revisión
}

\begin{abstract}
Resumen. Las dermatopatías parasitarias son enfermedades que representan aproximadamente el $30 \%$ del total de casos atendidos en hospitales y clínicas veterinarias de animales de compañía. Una de las patologías de la piel es la sarna, que es causada por un ácaro Sarcoptes scabie variedad canis. Altamente contagiosa entre los animales domésticos como perros con potencial zoonótico pudiendo infestar al ser humano. La enfermedad se manifiesta con prurito intenso en la piel, formando costras hemorrágicas, pérdida de pelos y provocando la aparición de heridas. Los exámenes de diagnóstico consisten en la prueba de arrancamiento del pelo, tricograma, raspado superficial y ensayo terapéutico con ectoparasiticida Varios productos antiparasitarios vienen siendo usados para el tratamiento de sarna sarcóptica que varían desde múltiples aplicaciones tópicas a la dosis única inyectable. Esta revisión objetivó verificar la importancia clínica en la identificación de los signos clínicos, formas de diagnósticos y estrategias de control parasitario apropiados y eficaces, de esta forma contribuyendo para mejor proteger a los perros de estos parásitos. A través del presente estudio, se nota la evidencia de la atención del médico veterinario a los signos clínicos del ácaro en la clínica de pequeños animales, su tratamiento debe comenzar tan temprano como el diagnóstico, pues, además del factor bienestar animal, se tiene sarna sarcóptica que se considera una zoonosis, siendo así un caso de salud pública.
\end{abstract}

Palabras clave: ácaro, perros, escabiosis, sarna sarcástica

\section{Introdução}

A dermatologia veterinária tem como função analisar e tratar animais diagnosticados com doenças de pele, visando um tratamento eficaz, dando ao animal uma melhor e mais satisfatória saúde e qualidade de vida. Dermopatias parasitárias são doenças cosmopolitas e estão presentes em $30 \%$ dos casos de pacientes analisados em clínicas e hospitais veterinários (Lillie, 1965; Mariath et al., 1995).

Uma das doenças de pele mais conhecidas é a sarna, uma dermatologia ocasionada por um ácaro denominado Sarcoptes scabiei variedade canis, parte do filo Arthropoda, da classe Arachnida ordem Acari, e família Sarcoptidae (ácaro escavadores) causando ao enfermo uma inflamação na pele ligada, ou não, a um grau de coceira, presença de crostas hemorrágicas, perda de pelo e aparecimento de feridas, causando incômodo ao animal. Além disso, é uma patologia parasitária altamente contagiosa entre os animais domésticos, de grande porte, ovinos, caprinos e roedores, por exemplo, ainda com perigo de parasitar o ser humano (Heukelbach \& Feldmeier, 2008; Piccinin et al., 2008; Urquhart, 1996). Os sinais clínicos dessa patogênese variam de acordo com a imunidade do hospedeiro. Pacientes cujo sistema imune é pouco resistente, desnutridos ou com deficiência de imunidade são mais susceptíveis, tendo em vista a dificuldade para desenvolver mecanismos para limitar o desenvolvimento desses organismos. Sabendo-se que, para a patologia da sarna não há vacinas, tem-se então a necessidade de pôr em prática a prevenção (Isola et al., 2012; Miller et al., 2013; Silva et al., 2013).

Como citado anteriormente, a doença se manifesta com prurido intenso na pele, seguida de crostas hemorrágicas, perda de pêlo e aparecimento de lesões. Estes organismos atacam, preferencialmente, as áreas onde a pelagem é mais espessa, como orelhas, focinho, cabeça e pescoço, por exemplo. A escavação do ácaro gera reações inflamatórias que levam ao endurecimento da pele, descamação, alopecia (perda de pêlo) e infecções secundárias. Alguns dos exames utilizados para se obter o diagnóstico são: tricograma, raspagem superficial e trial terapêutico com ectoparasiticida (Wall \& Shearer, 2001 (Ettinger \& Feldman, 2004; Piccinin et al., 2008).

Os ácaros devem ser vistos como organismos a serem exterminados o mais rápido possível, da mesma forma deve ser visto o tratamento para os diagnosticados. Já existem e são usados diversos produtos antiparasitários no tratamento da sarna sarcóptica, como aplicações múltiplas por via epidérmica, diretamente na pele, até dose única injetável (Argüello et al., 2001; Medleau et al., 2003; Patel \& Forsythe, 2011; Scott et al., 1996). O tratamento recomendado começa no banho dos cães, utilizando xampu anti-seborréico para eliminar as crostas, junto com a aplicação do escabicida tópico por todo o corpo, em intervalos de 7 dias durante, no mínimo, 5 semanas. Entre os produtos de via epidérmica indicados estão a solução de sulfeto de cálcio $2 \%$ a $3 \%$, organoclorados, organofosforados e 
Ivermectina, por exemplo. Levando em conta que esse organismo pode viver no ambiente em média de 21 dias, é indicada a limpeza frequente do ambiente, junto a aplicação de um pesticida (Medleau et al., 2003; Moriello, 2004).

Ao estudar os ácaros escavadores em cães através das produções científicas, esta revisão objetivou verificar a importância clínica na medicina veterinária através da identificação dos sinais clínicos, formas de diagnósticos e estratégias de controle parasitário apropriado e eficaz, dessa forma contribuindo para melhor proteger os cães destes parasitos.

\section{Morfologia e ciclo}

O gênero Sarcoptes, também conhecido como ácaros escavadores, por escavarem as galerias da pele (intradérmica), nas quais penetram profundamente, provocando um espessamento da pele. São ácaros de morfologia globosa, com rosto curto e largo, patas curtas e grossas e encaixadas total ou parcialmente no idiossima (corpo do ácaro) que apresenta áreas escamosas e espinhos curtos e grossos na face dorsal. Seu tamanho varia de 0,2 a $0,5 \mathrm{~mm}$, possui um gnatossoma cônico (tão longo quanto largo), pedicelo longo e não segmentado, e ânus terminal (Monteiro, 2011).

Os machos saem de sua galeria em direção à superfície em busca das fêmeas púberes, porém, assim que acasalam, morrem. Em relação à fêmea ovígera, cujo seu ovo tem um ciclo evolutivo tem duração de dez a quatorze dias, onde estes parasitas não mordem nem sugam o sangue, pois se alimentam de fluidos intercelulares. Já na fase de incubação, que dura em torno de três dias, cada ovo eclode uma larva hexápode, que são capazes de viver na galeria cavando ou perfurar uma nova galeria no animal (Farias, 2007; Ferrari et al., 2008; Zanon et al., 2008).

O parasita completa o seu ciclo (ovo - larva - ninfa - adulto) em três semanas. Os ácaros provocam a desorganização dos tecidos da pele escavando-a, gerando antígenos, (substância capaz de entrar no corpo e se ligar a seus anticorpos) que poderão causar irritabilidade à pele e, em seguida, reações alérgicas. Sabe-se que a sarna sarcóptica é altamente contagiosa e inicialmente transmitida pelo contato direto, porém, deve-se estar atento aos objetos de higiene, pois pode ser um meio de hospedar o parasita (Birchard et al., 1995; Guimarães et al., 2001).

\section{Importância clínica}

Os ácaros Sarcoptes scabiei tem como alvo regiões com pouco pelo, como axilas, cotovelo, peito, abdômen, tarsos, podendo generalizar-se. As lesões se classificam em primarias e secundarias, as primarias origina-se diretamente da patologia, já as secundárias desenvolvem-se numa fase posterior, devido à ação do paciente ou outros fatores externos (Miller et al., 2013; Scott et al., 1996).

Uma das características principais dessa parasitose é o prurido que aparece a noite, quando o hospedeiro vai dormir. Os excrementos dos ácaros são responsáveis pela reação de hipersensibilidade. Também se encontram a presença de alopecia, crostas, escoriações, e hiperemia. As crostas acometem mais a região do cotovelo, jarretes, dígitos e face (onde fica o primeiro lugar para se obter raspados diagnósticos) (Fourie et al., 2007; Monteiro, 2011).

Para precisão do diagnostico o raspado tem que ser observado em algum estágio do acaro. Deve-se procurar regiões não escoriadas, pápulas avermelhadas e que tenha presente crostas amareladas na superfície. Um ácaro, ovo ou pellets fecais ovais ratificam o diagnóstico (Patel \& Forsythe, 2011; Pinho et al., 2015; Scott et al., 1996). Castro et al. (2005) no período de 19 anos estabeleceu diagnóstico de escabiose em 3.623 cães (78,5\%), onde 1202 (53\%) eram machos e 1066 (47\%) eram fêmeas. Quanto ao padrão racial constatou-se 1321 (58\%) cães eram de raça definida. De acordo com Nóbrega (2018) em seu estudo feito a partir de levantamento de dados dos arquivos dos com cães do Hospital Veterinário da cidade de Areia, Paraíba, durante o período de 2012 a 2018, teve como objetivo do levantamento diagnosticar cães com dermatopatias provocadas por ácaros causadores de sarna, no qual foram submetidos a exames de pele e $15 \%$ dos cães foram diagnosticados com sarna sarcóptica. O mesmo estudo foi realizado por Lopes et al. (2018) em cães atendidos no Hospital Veterinário da FIMCA, Porto Velho, Rondônia, no período de outubro de 2017 a junho de 2018, onde a Sarcoptes sp. esteve em 5,3\% dos cães examinados. Pelo parâmetro de cães com padrão racial definido, no estudo de Nóbrega (2018) a maior incidência foram os animais com raça definida (53\%), contra $47 \%$ de casos de animais sem 
padrão racial definido, os mesmos valores oram encontrados em relação ao sexo, e resultados semelhantes foram obtidos por Lopes et al. (2018). Assim, evidenciando que esta doença não tem predileção racial, sexual ou idade. A doença é muito contagiosa pelo contato físico, geralmente afeta aos animais mais debilitados. Entretanto autores norte-americanos refiram uma maior tendência de acometimento de algumas raças, geralmente aquelas de pelagem longa (Matos et al., 2012; Sischo et al., 1989; Zur et al., 2002).

\section{Controle}

A forma de controle varia em função do paciente, proprietário e do clínico. Estão disponíveis planos terapêuticos diversos consoante a via de administração, via tópica por banhos (amitraz concentração, 0,025 a $0,06 \%$, off label, uma a duas vezes por semana; solução enxofre de cal a $2 \%$ semanalmente, durante 2 a 3 semanas consecutivas, desvantagem: mau odor, cora o pelo, vômitos e diarreia se ingerir) ou spot-on (selamectina, 6 a $12 \mathrm{mg} / \mathrm{kg}$ mensalmente, por 2 meses), subcutânea (ivermectina, off-label, $200-400 \mathrm{mg} / \mathrm{kg}$, atenção raças susceptíveis ou indivíduos com mutação no gene MRD1 (nome atual gene ABCB1)), oral (milbemicinaoxima, $2 \mathrm{mg} / \mathrm{kg} \mathrm{q} 7$ dias 4 a 6 tomas, mais dispendiosa e menos tóxica) (Andrade, 2008; Ferrari et al., 2008; Piccinin et al., 2008).

Ao manusear animais infectados devem-se ter cuidados e é recomendada a proteção (luvas, roupa descartável, entre outros) ao realizar o tratamento, pela facilidade de contração dessa doença de pele. $\mathrm{O}$ ambiente contaminado por ácaros deve ser higienizado totalmente e tratado com produtos acaricidas. Todos os animais também habitantes devem ser tratados simultaneamente (Ferrari et al., 2008).

Segundo Baltazar et al. (2004), os problemas relacionados à saúde animal e, consequentemente, à saúde pública podem ser minimizados quando se aplica a educação em saúde. Esses mesmos autores discorrem que para garantir uma relação homem e animal saudável é necessário educar as pessoas, não simplesmente com propagandas e divulgações em massa nos meios de comunicação, mas também com programas sistemáticos de educação em saúde, devidamente direcionados ao público alvo.

\section{Conclusão}

Através do presente estudo, nota-se, a evidência da atenção do médico veterinário aos sinais clínicos do ácaro na clínica de pequenos animais, seu tratamento deve começar tão mediatamente quanto o diagnóstico, pois, além do fator bem-estar animal, tem-se a sarna sarcóptica que é considerada uma zoonose, sendo assim um caso de saúde pública. $\mathrm{O}$ uso da Ivermectina ${ }^{\circledR}$ administrada por via oral em quatro doses com intervalo de sete dias juntamente com banhos tópico, se mostra eficaz no controle de Sarcoptes scabiei em cães naturalmente infestados.

\section{Referências bibliográficas}

Andrade, S. F. (2008). Manual da Terapêutica Veterinária. São Paulo: Rocca.

Argüello, M. R. H., Díez-Baños, N., Martínez-González, B. \& Rojo-Vazquez, F. A. (2001). Efficacy of moxidectin $1 \%$ injectable against natural infection of Sarcoptes scabiei in sheep. Veterinary Parasitology, 102(1-2):143-150.

Baltazar, C., Fernandes, I. B., Dias, R. A., Ferreira, F. \& Pinheiro, S. R. (2004). Formação de multiplicadores na área de saúde pública e higiene de alimentos. Revista Ciência em Extensão, 1(1):79-90.

Birchard, S. J., McLoughlin, M. A. \& Smeak, D. D. (1995). Chylothorax in the dog and cat: a review. Lymphology, 28(2):64-72.

Castro, R. C. C., Lucarts, L. E. B., Delayte, E. H., Otsuka, M., Germano, P. M. L. \& Larsson, C. E. (2005). Levantamento retrospectivo de casos de escabiose canina e felina, atendidos na Faculdade de Medicina Veterinária e Zootecnia da Universidade de São Paulo, no período compreendido entre 1984 e 2002. Brazilian Journal of Veterinary Research and Animal Science, 42(2):135-142.

Ettinger, S. \& Feldman, E. (2004). Tratado de medicina interna veterinária: doenças do cão e do gato. Rio de Janeiro: Guanabara Koogan.

Farias, M. R. (2007). Dermatite atópica canina: da fisiopatologia ao tratamento. Clínica Veterinária, 6948-62.

Ferrari, M. L., Prado, O. P. \& Spigolon, M. O. Z. (2008). Sarna sarcóptica em cães. Revista Científica Eletrônica de Medicina Veterinária, 6(10):1-5. 
Fourie, L. J., Kok, D. J., Du Plessis, A. \& Rugg, D. (2007). Efficacy of a novel formulation of metaflumizone plus amitraz for the treatment of demodectic mange in dogs. Veterinary Parasitology, 150(3):268-274.

Guimarães, J. H., Battesti, D. M. B. \& Tucci, E. C. (2001). Ectoparasitos de importância veterinária. São Paulo: FAPESP.

Heukelbach, J. \& Feldmeier, H. (2008). Epidemiological and clinical characteristics of hookwormrelated cutaneous larva migrans. The Lancet Infectious Diseases, 8(5):302-309.

Isola, J. G. M. P., Cadioli, F. A. \& Nakage, A. P. (2012). Erliquiose canina-revisão de literatura. Revista Científica Eletrônica de Medicina Veterinária, 181-11.

Lillie, R. D. (1965). Histopathologic technic and practical histochemistry. New York, USA: McGrawHill Book Company.

Lopes, T. V., Souza, T. A., Reis, L. T. P., Morais, W. E. S., Oliveira, V. C. \& Pimenta, C. N. (2018). Prevalência de dermatites causadas por ácaros em cães e gatos atendidos no hospital veterinário da fimca, Porto Velho/RO. Paper presented at the Anais $1^{\circ}$ Congresso Brasileiro da Anclivepa, Anclivepa, Roraima, Brasil.

Mariath, J. E. A., Coelho, G. C., Santos, R. P., Heuser, E. D., Ayub, D. M. \& Cocucci, A. E. (1995). Aspectos anatômicos e embriológicos em espécies do gênero Ilex. In H. Winge, A. G. Ferreira, J. E. A. Mariath \& L. C. Tarasconi (Eds.), Erva-mate: Biologia e cultura no cone sul (pp. 263-280). Porto Alegre, Rio Grande do Sul: Editora da Universidade Federal do Rio Grande do Sul.

Matos, C. B., Madrid, I. M., Santin, R., Azambuja, R. H., Schuch, I., Meireles, M. C. A. \& Cleff, M. B. (2012). Multifactorial dermatitis in a dog. Arquivo Brasileiro de Medicina Veterinária e Zootecnia, 64(6):1478-1482.

Medleau, L., Hnilica, K. A. \& Fagliari, G. S. (2003). Dermatologia de pequenos animais: atlas colorido e guia terapêutico. São Paulo: Roca.

Miller, W. H., Griffin, C. E., Campbell, K. L. \& Muller, G. H. (2013). Muller and Kirk's Small Animal Dermatology. Philadelphia, USA: Elsevier Health Sciences.

Monteiro, S. G. (2011). Parasitologia na medicina veterinária (Vol. 1). São Paulo: Roca.

Moriello, K. A. (2004). Treatment of dermatophytosis in dogs and cats: review of published studies. Veterinary Dermatology, 15(2):99-107.

Nóbrega, B. G. (2018). Estudo retrospectivo de demodicose e escabiose em cães atendidos no Hospital Veterinário de Areia - PB, Campus II Areia, Paraíba: Universidade Federal da Paraíba.

Patel, A. \& Forsythe, P. J. (2011). Dermatologia em pequenos animais. Rio de Janeiro: Elsevier Brasil.

Piccinin, A., Ferrari, M. L. O. P., O., P. M. \& Spigolon, Z. (2008). Sarna sarcóptica em cães. Revista Científica Eletrônica de Medicina Veterinária, 7(10):1679-7353.

Pinho, R. M., Monzón, M. F. \& Simões, J. (2015). Dermatologia veterinária em animais de companhia.

Scott, D. W., Muller, G. H. \& Kirk, R. W. (1996). Dermatologia dos pequenos animais (Vol. 1). Rio de Janeiro: Interlivros.

Silva, M. V. M., Fernandes, R. A., Nogueira, J. L. \& Ambrósio, C. E. (2013). Erliquiose canina: revisão de literatura. Arquivos de Ciências Veterinárias e Zoologia da UNIPAR, 14(2):139-143.

Sischo, W. M., Ihrke, P. J. \& Franti, C. E. (1989). Regional distribution of ten common skin diseases in dogs. Journal of the American Veterinary Medical Association, 195(6):752-756.

Urquhart, G. M. (1996). Parasitologia veterinária (2 ed.). Rio de Janeiro: Guanabara Koogan.

Zanon, J. P., Gomes, L. A., Cury, G. M. M., Teles, T. C. \& Bicalho, A. P. C. V. (2008). Dermatite atópica canina. Semina: Ciências Agrárias, 29(4):905-920.

Zur, G., Ihrke, P. J., White, S. D. \& Kass, P. H. (2002). Canine atopic dermatitis: a retrospective study of 266 cases examined at the University of California, Davis, 1992-1998. Part I. Clinical features and allergy testing results. Veterinary Dermatology, 13(2):89-102.

Recebido: 30 de maio, 2019.

Aprovado: 30 de junho, 2019.

Publicado: 7 de agosto, 2019.

Licenciamento: Este artigo é publicado na modalidade Acesso Aberto sob a licença Creative Commons Atribuição 4.0 (CC-BY 4.0), a qual permite uso irrestrito, distribuição, reprodução em qualquer meio, desde que o autor e a fonte sejam devidamente creditados. 